\section{Bicuspid aortic valve associated aortopathy: a genetic disease}

\author{
Gibran Minero, ${ }^{1}$ Simon C. Body ${ }^{2}$ \\ 'Harvard Medical School, Boston, MA; \\ 2Department of Anesthesiology, \\ Perioperative and Pain Medicine, Brigham \\ and Women's Hospital, Harvard Medical \\ School, Boston, MA, USA
}

\begin{abstract}
As the most common congenital heart defect, understanding the etiology and progression of aortopathy in bicuspid aortic valve (BAV) is imperative to management of patients with BAV. A reasonable hypothesis, based on the strength of evidence for both genetic and hemodynamic causes of BAV-associated thoracic aortic disease (TAD), is that BAV is caused by genetic variant(s) that also predispose to TAD by a common mechanism; presumably by cell-signaling resulting in an embryologic defect that causes BAV and a postnatal risk of TAD that is accentuated by hemodynamic stress of abnormal flow through the $\mathrm{BAV}$ valve. Clinical heterogeneity seen in BAVassociated TAD is likely due to individual genetic variation and the severity of hemodynamic alteration.
\end{abstract}

\section{Introduction}

Controversy exists in the literature regarding the cause(s) of bicuspid aortic valve-associated thoracic aortic disease (TAD); specifically whether it is hemodynamic in origin, or whether there are genetic causes that predispose to TAD in patients with bicuspid aortic valve (BAV). The increased aortic wall stress of chronic hypertension, smoking and age-related changes in aortic wall biology are risk factors for TAD and its morbidity and mortality. ${ }^{1,2}$ Similarly, the long-known relationship between aortic stenosis and post-stenotic dilation of the thoracic aorta further emphasizes the importance of aortic wall stress upon development of TAD. However, it is clear that TAD is an inherited disease that occurs without apparent hemodynamic etiology. ${ }^{3,4} \mathrm{BAV}$ is associated with an order of magnitude increase in the lifetime prevalence of TAD to $\sim 50 \%,{ }^{5}$ most commonly presenting as asymmetric dilation of the ascending aorta beyond the sinotubular junction, and a far-greater relative risk in younger age groups. ${ }^{6}$ Yet not all patients with BAV will develop TAD, regardless of the severity of valve dysfunction.
The important unanswered questions surrounding TAD occurring in BAV disease are: are there specific mechanisms of TAD that occur only from the hemodynamic consequences of abnormal flow and aortic wall stress from a normally functioning bicuspid valve, and are there specific mechanisms for TAD that arise from genetic variation that either caused the bicuspid valve or are independent of the etiology of the bicuspid valve. These questions are important to the management of patients with BAV, notably for directing research for prevention or delaying the onset of $\mathrm{TAD}$; to determine monitoring of TAD by either imaging or biomarker measurements; and for surgical management of the dilating aorta.

\section{The aortic valve and aorta have common ectodermal and mesodermal origins}

The aortic valve and aorta have common progenitor origins that underscore their propensity for concurrent congenital disease. Key molecular signaling pathways are responsible for proliferation and migration of contributing cell populations and genetic and epigenetic variation in these signaling pathways contribute to congenital heart defects.

The embryonic heart initially forms as a linear tube from first heart field progenitor cells with the primordial ventricle emptying into a single outflow tract (OFT) - the truncus arteriosus. The OFT, aortic valve and ascending aorta are formed from two populations of interdependent cells from: i) second heart field (SHF) progenitor cells arising from a pharyngeal mesodermal clonal population that also contributes to the right ventricle; ii) cardiac neural crest (CNC) cells arising from somites 1-3 of the dorsal neural crest that migrate through the pharyngeal arches into the aorta and distal OFT. The OFT is elongated during the fifth week of gestation by migrating SHF cells, forming bulbar ridges.

CNC cells contribute to the outflow tracts, aortic and pulmonary valves and the smooth muscle and connective tissue of the aorta, from the ascending aorta to the site of aortic coarctation. CNCs migrate into the bulbar ridges to form the helical aortic-pulmonary septum that fuses during the sixth gestational week, allowing for separation of the aorta and pulmonary trunk. CNCs orchestrate aortic and pulmonary valve development by CNC invasion of the endothelial cells of the primitive endocardial cushions to form recognizable thin aortic valve leaflets. ${ }^{7,8}$ Despite considerable contribution of the CNC to the maturation of the endocardial cushions into semi-lunar valves,
Correspondence: Simon C. Body, Department of Anesthesiology, Perioperative and Pain Medicine, Brigham and Women's Hospital, Harvard Medical School, 75 Francis St, Boston, MA 02115, USA. Tel.: 001.617.732.7330 - Fax: 001.617.730.2813. E-mail: sbody@partners.org

Key words: aortic valve, aorta, aneurysm, genetic, cardiac neural crest.

Funding: this work was supported by NIH grant R01HL114823.

Received for publication: 3 December 2014.

Revision received: 2 February 2015.

Accepted for publication: 2 February 2015.

This work is licensed under a Creative Commons Attribution NonCommercial 3.0 License (CC BYNC 3.0).

(C) Copyright G. Minero and S.C. Body, 2014 Licensee PAGEPress, Italy

Cardiogenetics 2014; 4:4857

doi:10.4081/cardiogenetics.2014.4857

few CNC-derived cells are recognizable in the mature semilunar valve leaflets. ${ }^{9}$ More likely, CNC cells drive SHF matrix production and subsequent apoptosis. ${ }^{10}$ An example is association between NOTCH1 mutations and BAV. ${ }^{11}$ Notch signaling regulates endothelial-mesenchymal transformation during endocardial cushion formation, ${ }^{12}$ perhaps by regulation of Fgf8 signaling. ${ }^{13}$ Deficiencies in Fgf8 can cause BAV and aortic smooth muscle abnormalities. ${ }^{14}$ The commonality of CNC in aortic valve and aortic development readily leads to the hypothesis that abnormalities of CNC proliferation, migration or signaling are responsible for the spectrum of BAV and TAD.

\section{Bicuspid aortic valve is a polygenic abnormality}

BAV is the most common congenital valvular abnormality, occurring in $0.5-1.2 \%$ of the population, more commonly in males and those with Turner syndrome, ${ }^{15}$ and leads to greater morbidity and mortality than all other congenital heart defects combined. ${ }^{16} \mathrm{BAV}$ is an inherited disorder with $\sim 9 \%$ prevalence in firstdegree relatives but a variable inheritance pattern. ${ }^{15,17,18}$ The difficulty in dissecting out the genetic causes of BAV, that may or may not also cause TAD, is because highly-penetrant singlegene inheritance is observed in only a few well-reported families. ${ }^{11}$ Far more commonly, the inheritance pattern is unclear with few individuals in a family exhibiting $\mathrm{BAV}$ or TAD, indicating polygenic disease perhaps with a cumulative burden of lower-risk common and 
uncommon variants in the etiology of BAV. To date, rare variants or haploinsufficiency of NOTCH1, GATA4/5/6, NOS3 and other genes have been associated with BAV in mice and humans. These genes have key signaling roles in both SHF and CNC migration and cell-signaling and are likely causative of BAV. ${ }^{11,19-24}$

\section{Thoracic aortic disease is a polygenic disease}

An increasing list of common and uncommon genetic variants have been demonstrated to be associated with $\mathrm{TAD}$, including common variants in $F B N 1,25$ and uncommon or rare variants in ACTA2, FBN1, FLNA, MYH11, SMAD3 and TGFBR1/2, amongst others. ${ }^{5,26}$ Adding to the evidence for a genetic role in TAD, especially in $\mathrm{BAV}$, tricuspid aortic valve (TAV) first-degree relatives of a BAV patient may have a higher rate of TAD and lower aortic distensibility than the population average but this observation is in question..$^{27,28}$ Histologic abnormalities including medial degeneration and elastin fragmentation have been described in both the non-dilated and dilated ascending aorta of BAV patients, implying a common mechanism of TAD. ${ }^{29}$ Other clinical observations that less-rigorously reinforce a genetic contribution to TAD in BAV are the occurrence of TAD in BAV patients without significant aortic stenosis especially at young age,$^{30}$ or following aortic valve replacement, ${ }^{31}$ that average aortic size is greater in patients with BAV than TAV, even after controlling for hemodynamic effects of valvular defects $^{32}$ and finally, the severity and prevalence of aortopathy in patients with BAV is greater than what would be expected based on severity of aortic stenosis or regurgitation alone. ${ }^{17,29}$

\section{A normally functioning bicuspid valve causes abnormal and unequal wall stress in the ascending aorta}

4D magnetic resonance imaging of the normal TAV shows blood to the ascending aorta with a moderate helical flow in line with the axis of the aorta and with low wall shear stress. ${ }^{33}$ Asymmetric opening of the BAV leads to turbulent, non-axial flow in the ascending aorta with a majority of BAV patients having markedlyincreased right handed helical flow. ${ }^{34}$ This results in increased an unequal wall stress on the ascending aorta ${ }^{34,35}$ and sometimes, to asymmetric dilation of the aortic root and ascending aorta. ${ }^{36-39}$ Leaflet fusion sub-type is associated with dilatation pattern of the aorta - patients with right-left cusp fusion had larger aortic root diam-

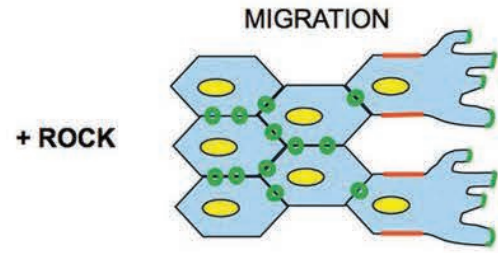

CONDENSATION
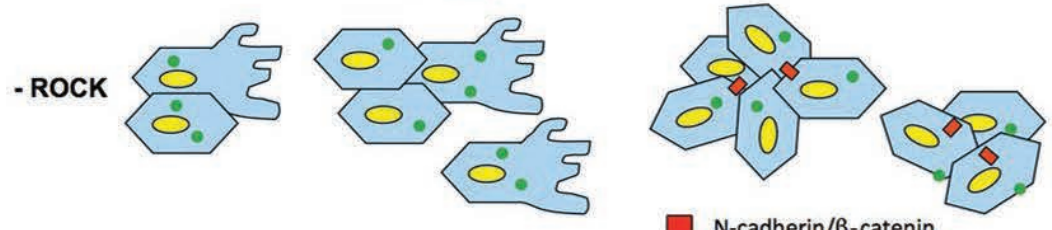

$\mathrm{N}$-cadherin/ $\beta$-catenin

Connexin-43

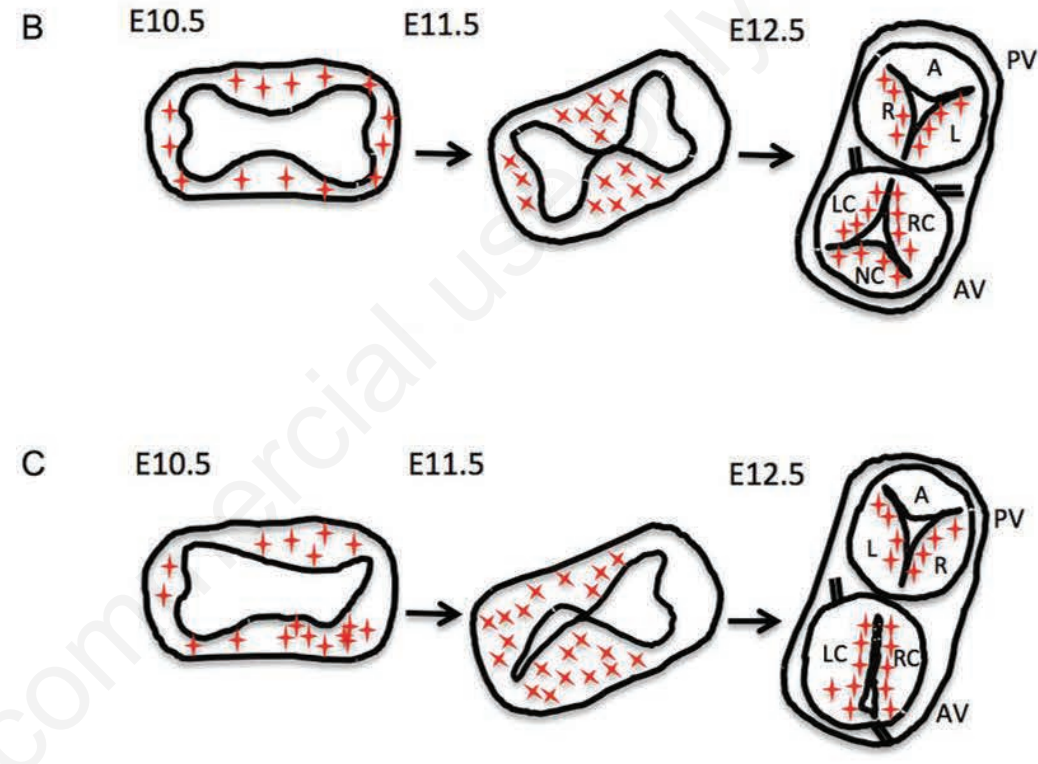

D

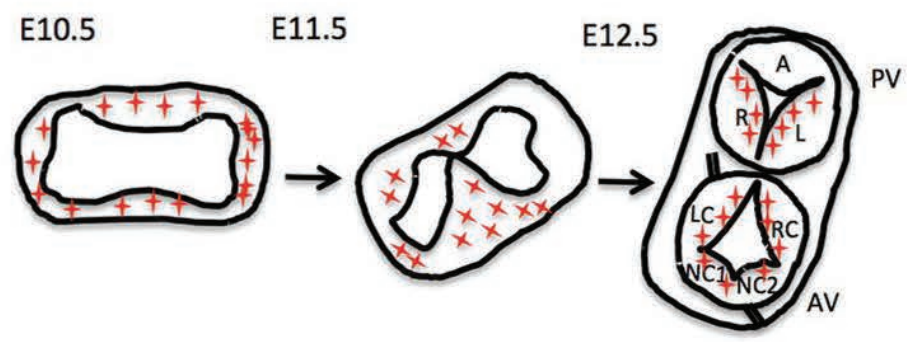

Figure 1. Model for abnormal aortic and pulmonary valve patterning in the developing embryo. A) Abnormalities in cardiac neural crest (CNC) cell adhesion and/or signaling results in disrupted CNC condensation in the outflow cushions. B) Distribution of CNC (red stars) in the outflow tract in normal embryos as the outflow tract septates and tricuspid aortic and pulmonary valves are formed. C and D) CNC do not condense normally in mutant embryos. As a consequence the valve leaflets are abnormally positioned and irregularly sized yielding a bicuspid aortic phenotype (C) or a quadricuspid phenotype (D). From Philips HM et al. Cardiovasc Res 2013;99:452-60 (Open Access). 
eter and were younger when presenting for surgical interventions than those with right-noncoronary fusion. ${ }^{39,40}$

\section{Responses to abnormal aortic wall stress are not the same in bicuspid and tricuspid aortic valve patients}

There are conflicting data on the aortic wall responses to abnormal wall stress in bicuspid and tricuspid aortic valve patients. There are both methodological and interpretative issues that drive the issue. But lets establish a few points over which there is little controversy.

The response of the ascending aorta to increased wall stress has different cellular mechanisms for BAV and TAV patients. In BAV-associated TAD, smooth muscle apoptosis appears to be the dominant histologic event. In contrast, TAVassociated TAD is characterized by elastic fragmentation, cystic medial necrosis and fibrosis, and inflammation. ${ }^{41}$ These observations are not confined to the aorta - they are also seen in pulmonary artery of BAV patients, emphasizing the importance of the common aortopulmonary trunk in embryogenesis. ${ }^{42}$ Complicating this are differences in the patterns of extracellular matrix proteins seen between the concave and convex surfaces of the ascending aorta of BAV patients. ${ }^{43}$

When the BAV is replaced, without aortic replacement, reducing aortic wall stress, the average rate of aortic wall dilation returns to low levels seen in TAV patients. ${ }^{44,45}$ This is not surprising as the aortic response to lower wall stress is likely to be reduced after aortic valve replacement (AVR) and the average rate should be fairly similar. But we are not treating average patients; rather it is the outliers that count. Common genetic variants likely have some effect on the average rate of aortic dilation but when aortic wall stress is reduced the impact of these common variants is likely to be small. We can prudently hypothesize that some patients with a burden of common and rare variants that cause aortic dilation will proceed with aortic dilation above the average rate and may require later aortic replacement or repair. This hypothesis is important as it may direct management of the patient presenting for AVR with a moderately dilated aorta. But this hypothesis needs to be proven; which can only be done with sufficiently wellpowered and well-phenotyped cohorts with collected DNA, aortic valve and aortic tissues. Indeed, in the absence of these data we can say that the only predictors of rapid aortic dilation are young age, male gender, the aortic root phenotype including the conjoint cusp opening angle, i.e. alignment of the fused cusp to the aortic axis and right-left BAV configuration. ${ }^{46-48}$

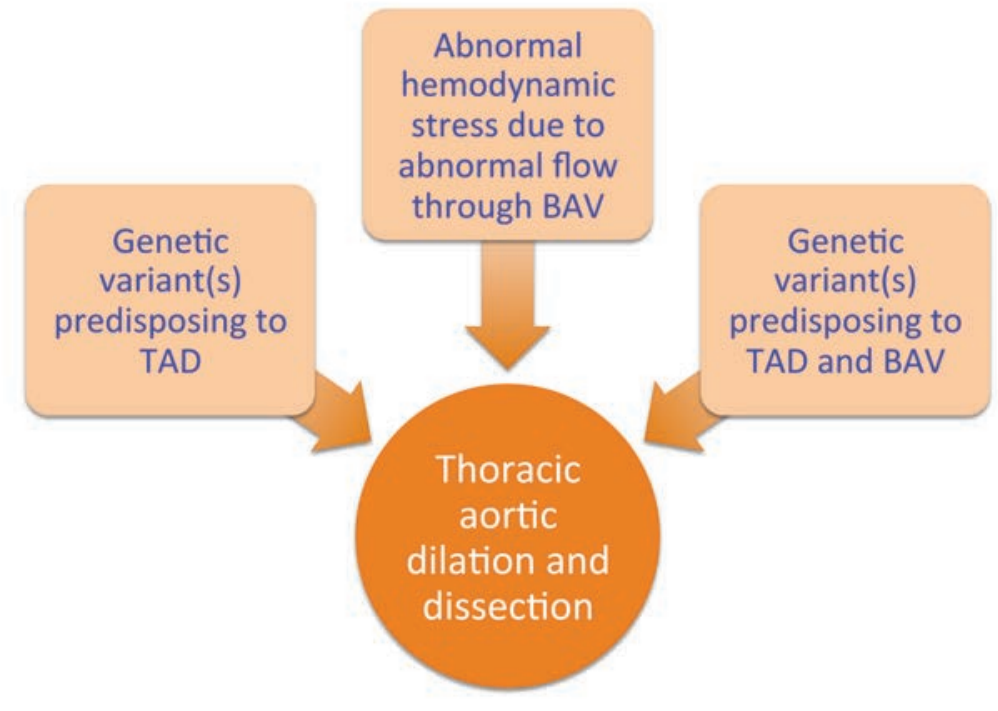

Figure 2. A hypothesis of interacting genetic and hemodynamic causes of bicuspid aortic valve (BAV)-associated thoracic aortic disease (TAD). Common and rare genetic mutations cause BAV and TAD that predispose to TAD in the presence of normal and abnormal hemodynamic stresses from the BAV. Combinations of genetic mutation, BAV configuration and aortic wall shear stress yield different TAD phenotypes between individuals.

\section{How abnormal aortic wall stress and an abnormal aorta interact to cause thoracic aortic disease}

While cardiovascular risk factors and older age contribute to TAD, genetic variants in FBN1, ACTA2, FLNA, MYH11, SMAD3, and TGFBR1/2 are also associated with TAD in non-BAV patients. Thus far, only common variants in FBN1 have been associated with TAD in BAV and reduced fibrillin-1 protein is seen even in patients with normal BAV function. ${ }^{49}$ There is marked phenotypic variability in wall stress and TAD even amongst patients with specific BAV fusion pattern and similar levels of valve dysfunction..$^{50}$ It is reasonable to believe that varying genetic and non-genetic characteristics between individuals contribute to the level of TAD seen in each individual. Studying these pathological flow patterns and combining it with protein expression and genetic risk profiles could lead to more optimal risk assessment and treatment guidelines. However, despite progress in understanding the varied contributing etiologies in BAV-associated $\mathrm{TAD}$, we still do not fully understand how these factors interact to accurately predict the progression of TAD development or phenotypic expression of TAD.

\section{References}

1. Della Corte A, Bancone C, Quarto C, et al. Predictors of ascending aortic dilatation with bicuspid aortic valve: a wide spectrum of disease expression. Eur $\mathrm{J}$ Cardiothorac Surg 2007;31:397-404; discussion -5.

2. Thanassoulis G, Yip JW, Filion K, et al. Retrospective study to identify predictors of the presence and rapid progression of aortic dilatation in patients with bicuspid aortic valves. Nat Clin Pract Cardiovasc Med 2008;5:821-8.

3. Lemaire SA, McDonald ML, Guo DC, et al. Genome-wide association study identifies a susceptibility locus for thoracic aortic aneurysms and aortic dissections spanning FBN1 at 15q21.1. Nat Genet 2011;43:9961000.

4. Guo DC, Pannu H, Tran-Fadulu V, et al. Mutations in smooth muscle alpha-actin (ACTA2) lead to thoracic aortic aneurysms and dissections. Nat Genet 2007;39:1488-93.

5. Bisleri G, Bagozzi L, Muneretto C. Current evidence and insights about genetics in thoracic aorta disease. Sci World J 2013;2013:962097.

6. Tzemos N, Therrien J, Yip J, et al. Outcomes in adults with bicuspid aortic valves. JAMA 2008;300:1317-25.

7. Combs MD, Yutzey KE. Heart valve development: regulatory networks in development and disease. Circ Res 2009;105:408-21.

8. Butcher JT, Markwald RR. Valvulogenesis: the moving target. Philos Trans R Soc Lond B Biol Sci 2007;362:1489-503.

9. Jiang X, Rowitch DH, Soriano P, et al. Fate of the mammalian cardiac neural crest. Development 2000;127:1607-16.

10. Jain R, Engleka KA, Rentschler SL, et al. Cardiac neural crest orchestrates remodeling and functional maturation of mouse semilunar valves. J Clin Invest 2011;121:422-30. 
11. Garg V, Muth AN, Ransom JF, et al. Mutations in NOTCH1 cause aortic valve disease. Nature 2005;437:270-4.

12. Timmerman LA, Grego-Bessa J, Raya A, et al. Notch promotes epithelial-mesenchymal transition during cardiac development and oncogenic transformation. Genes Dev 2004;18:99-115.

13. High FA, Jain R, Stoller JZ, et al. Murine Jagged1/Notch signaling in the second heart field orchestrates Fgf8 expression and tissue-tissue interactions during outflow tract development. J Clin Invest 2009;119:1986-96.

14. Brown CB, Wenning JM, Lu MM, et al. Cremediated excision of Fgf8 in the Tbx1 expression domain reveals a critical role for Fgf8 in cardiovascular development in the mouse. Dev Biol 2004;267:190-202.

15. Bondy C, Bakalov VK, Cheng C, et al. Bicuspid aortic valve and aortic coarctation are linked to deletion of the $\mathrm{X}$ chromosome short arm in Turner syndrome. J Med Genet 2013;50:662-5.

16. Ward C. Clinical significance of the bicuspid aortic valve. Heart 2000;83:81-5.

17. Girdauskas E, Borger MA, Secknus MA, et al. Is aortopathy in bicuspid aortic valve disease a congenital defect or a result of abnormal hemodynamics? A critical reappraisal of a one-sided argument. Eur J Cardiothorac Surg 2011;39:809-14.

18. Verma S, Siu SC. Aortic dilatation in patients with bicuspid aortic valve. N Engl J Med 2014;370:1920-9.

19. Bonachea EM, Chang SW, Zender G, et al. Rare GATA5 sequence variants identified in individuals with bicuspid aortic valve. Pediatr Res 2014;76:211-6.

20. Bonachea EM, Zender G, White P, et al. Use of a targeted, combinatorial next-generation sequencing approach for the study of bicuspid aortic valve. BMC Med Genomics 2014;7:56.

21. Foffa I, Ait Ali L, Panesi P, et al. Sequencing of NOTCH1, GATA5, TGFBR1 and TGFBR2 genes in familial cases of bicuspid aortic valve. BMC Med Genet 2013;14:44.

22. McKellar SH, Tester DJ, Yagubyan M, et al. Novel NOTCH1 mutations in patients with bicuspid aortic valve disease and thoracic aortic aneurysms. J Thorac Cardiovasc Surg 2007;134:290-6.

23. Padang R, Bagnall RD, Richmond DR, et al. Rare non-synonymous variations in the transcriptional activation domains of GATA5 in bicuspid aortic valve disease. J Mol Cell Cardiol 2012;53:277-81.

24. Thomas PS, Sridurongrit S, Ruiz-Lozano P, Kaartinen V. Deficient signaling via Alk2 (Acvr1) leads to bicuspid aortic valve development. PLoS One 2012;7:e35539.

25. LeMaire SA, Russell L. Epidemiology of tho- racic aortic dissection. Nat Rev Cardiol 2011;8:103-13.

26. Abdulkareem N, Smelt J, Jahangiri M. Bicuspid aortic valve aortopathy: genetics, pathophysiology and medical therapy. Interact Cardiovasc Thorac Surg 2013;17:554-9.

27. Biner S, Rafique AM, Ray I, et al. Aortopathy is prevalent in relatives of bicuspid aortic valve patients. $\mathrm{J}$ Am Coll Cardiol 2009;53:2288-95.

28. Loscalzo ML, Goh DL, Loeys B, et al. Familial thoracic aortic dilation and bicommissural aortic valve: a prospective analysis of natural history and inheritance. Am J Med Genet A 2007;143:1960-7.

29. Tadros TM, Klein MD, Shapira OM. Ascending aortic dilatation associated with bicuspid aortic valve: pathophysiology, molecular biology, and clinical implications. Circulation 2009;119:880-90.

30. Fernandes S, Khairy P, Graham DA, et al. Bicuspid aortic valve and associated aortic dilation in the young. Heart 2012;98:1014-9.

31. Yasuda H, Nakatani S, Stugaard M, et al. Failure to prevent progressive dilation of ascending aorta by aortic valve replacement in patients with bicuspid aortic valve: comparison with tricuspid aortic valve. Circulation 2003;108:Ii291-4.

32. Keane MG, Wiegers SE, Plappert T, et al. Bicuspid aortic valves are associated with aortic dilatation out of proportion to coexistent valvular lesions. Circulation 2000;102: lii35-9.

33. Lorenz R, Bock J, Barker AJ, et al. 4D flow magnetic resonance imaging in bicuspid aortic valve disease demonstrates altered distribution of aortic blood flow helicity. Magn Reson Med 2014;71:1542-53.

34. Bissell MM, Hess AT, Biasiolli L, et al. Aortic dilation in bicuspid aortic valve disease: flow pattern is a major contributor and differs with valve fusion type. Circ Cardiovasc Imaging 2013;6:499-507.

35. Lamata P, Pitcher A, Krittian S, et al. Aortic relative pressure components derived from four-dimensional flow cardiovascular magnetic resonance. Magn Reson Med 2014;72: 1162-9.

36. Cotrufo M, Della Corte A. The association of bicuspid aortic valve disease with asymmetric dilatation of the tubular ascending aorta: identification of a definite syndrome. J Cardiovasc Med (Hagerstown) 2009;10:291-7.

37. den Reijer PM, Sallee D, 3rd, van der Velden P, et al. Hemodynamic predictors of aortic dilatation in bicuspid aortic valve by velocityencoded cardiovascular magnetic resonance. J Cardiovasc Magn Reson 2010;12:4.

38. Hope MD, Hope TA, Meadows AK, et al.
Bicuspid aortic valve: four-dimensional MR evaluation of ascending aortic systolic flow patterns. Radiology 2010;255:53-61.

39. Russo CF, Cannata A, Lanfranconi M, et al. Is aortic wall degeneration related to bicuspid aortic valve anatomy in patients with valvular disease? J Thorac Cardiovasc Surg 2008;136:937-42.

40. Schaefer BM, Lewin MB, Stout KK, et al. Usefulness of bicuspid aortic valve phenotype to predict elastic properties of the ascending aorta. Am J Cardiol 2007;99: 686-90.

41. Balistreri CR, Pisano C, Candore G, et al. Focus on the unique mechanisms involved in thoracic aortic aneurysm formation in bicuspid aortic valve versus tricuspid aortic valve patients: clinical implications of a pilot study. Eur J Cardiothorac Surg 2013; 43:e180-6.

42. de Sa M, Moshkovitz Y, Butany J, David TE. Histologic abnormalities of the ascending aorta and pulmonary trunk in patients with bicuspid aortic valve disease: clinical relevance to the ross procedure. J Thorac Cardiovasc Surg 1999;118:588-94.

43. Cotrufo M, Della Corte A, De Santo LS, et al. Different patterns of extracellular matrix protein expression in the convexity and the concavity of the dilated aorta with bicuspid aortic valve: preliminary results. J Thorac Cardiovasc Surg 2005;130:504-11.

44. Charitos EI, Stierle U, Petersen M, et al. The fate of the bicuspid valve aortopathy after aortic valve replacement. Eur J Cardiothorac Surg 2014;45:e128-35.

45. Andrus BW, O'Rourke DJ, Dacey LJ, Palac RT. Stability of ascending aortic dilatation following aortic valve replacement. Circulation 2003;108:Ii295-9.

46. Della Corte A. Phenotypic heterogeneity of bicuspid aortopathy: a potential key to decode the prognosis? Heart 2014;100:96-7.

47. Folkersen L, Wagsater D, Paloschi V, et al. Unraveling divergent gene expression profiles in bicuspid and tricuspid aortic valve patients with thoracic aortic dilatation: the ASAP study. Mol Med 2011;17:1365-73.

48. Della Corte A, Bancone C, Conti CA, et al. Restricted cusp motion in right-left type of bicuspid aortic valves: a new risk marker for aortopathy. J Thorac Cardiovasc Surg 2012;144:360-9, 9 el.

49. Fedak PW, de Sa MP, Verma S, et al. Vascular matrix remodeling in patients with bicuspid aortic valve malformations: implications for aortic dilatation. J Thorac Cardiovasc Surg 2003;126:797-806.

50. Barker AJ, Markl M, Burk J, et al. Bicuspid aortic valve is associated with altered wall shear stress in the ascending aorta. Circ Cardiovasc Imaging 2012;5:457-66. 